\title{
A New Result on the Almost Increasing Sequences
}

\section{Hüseyin Bor*}

P. O. Box 121, TR-06502 Bahçelievler, Ankara, Turkey

Received: 25 May. 2013, Revised: 8 Sep. 2013, Accepted: 9 Sep. 2013

Published online: 1 Jan. 2014

Abstract: In this paper, we generalize a known theorem dealing with $|C, 1|_{k}$ summability factors to the $|C, \alpha, \beta|_{k}$ summability factors of infinite series. This theorem also includes some known and new results.

Keywords: Almost increasing sequences, Cesàro mean, absolute summability, infinite series, Hölder inequality, Minkowski inequality.

\section{Introduction}

A positive sequence $\left(b_{n}\right)$ is said to be an almost increasing sequence if there exists a positive increasing sequence $\left(c_{n}\right)$ and two positive constants $A$ and $B$ such that $A c_{n} \leq b_{n} \leq$ $B c_{n}$ (see [1]). Let $\sum a_{n}$ be a given infinite series. We denote by $t_{n}^{\alpha, \beta}$ the $n$th Cesàro mean of order $(\alpha, \beta)$, with $\alpha+\beta>$ -1 , of the sequence $\left(n a_{n}\right)$, that is (see [3])

$$
t_{n}^{\alpha, \beta}=\frac{1}{A_{n}^{\alpha+\beta}} \sum_{v=1}^{n} A_{n-v}^{\alpha-1} A_{v}^{\beta} v a_{v},
$$

where

$A_{n}^{\alpha+\beta}=O\left(n^{\alpha+\beta}\right), A_{0}^{\alpha+\beta}=1$ and $A_{-n}^{\alpha+\beta}=0$ for $n>0$.

The series $\sum a_{n}$ is said to be summable $|C, \alpha, \beta|_{k}, k \geq 1$, if (see [4])

$$
\sum_{n=1}^{\infty} \frac{1}{n}\left|t_{n}^{\alpha, \beta}\right|^{k}<\infty .
$$

If we take $\beta=0$, then $|C, \alpha, \beta|_{k}$ summability reduces to $|C, \alpha|_{k}$ summability (see [5]).

\section{The known result}

Theorem A ([ [7]). Let $\left(\varphi_{n}\right)$ be a positive sequence and $\left(X_{n}\right)$ be an almost increasing sequence. If the conditions

$\sum_{n=1}^{\infty} n\left|\Delta^{2} \lambda_{n}\right| X_{n}<\infty$

$\left|\lambda_{n}\right| X_{n}=O(1)$ as $n \rightarrow \infty$,
$\varphi_{n}=O(1)$ as $n \rightarrow \infty$,

$n \Delta \varphi_{n}=O(1)$ as $n \rightarrow \infty$,

$\sum_{v=1}^{n} \frac{\left|t_{v}\right|^{k}}{v X_{v}^{k-1}}=O\left(X_{n}\right)$ as $n \rightarrow \infty$

are satisfied, then the series $\sum a_{n} \lambda_{n} \varphi_{n}$ is summable $|C, 1|_{k}$, $k \geq 1$.

\section{The main result}

The aim of this paper is to generalize Theorem $\mathrm{A}$ in the following form.

Theorem. Let $\left(\varphi_{n}\right)$ be a positive sequence and $\left(X_{n}\right)$ be an almost increasing sequence. If the conditions (4), (5), (6) and (7) are satisfied and the sequence $\left(w_{n}^{\alpha, \beta}\right)$ defined by

$w_{n}^{\alpha, \beta}=\left\{\begin{array}{c}\left|t_{n}^{\alpha, \beta}\right|, \quad \alpha=1, \beta>-1 \\ \max _{1 \leq v \leq n}\left|t_{v}^{\alpha, \beta}\right|, 0<\alpha<1, \beta>-1\end{array}\right.$

satisfies the condition

$\sum_{v=1}^{n} \frac{\left(w_{v}^{\alpha, \beta}\right)^{k}}{v X_{v}^{k-1}}=O\left(X_{n}\right)$ as $n \rightarrow \infty$,

then the series $\sum a_{n} \lambda_{n} \varphi_{n}$ is summable $|C, \alpha, \beta|_{k}, 0<\alpha \leq$ $1,(\alpha+\beta-1)>0$ and $k \geq 1$.

We need the following lemmas for the proof of our theorem

Lemma 1 ( [2]). If $0<\alpha \leq 1, \beta>-1$ and $1 \leq v \leq n$, then

$$
\left|\sum_{p=0}^{v} A_{n-p}^{\alpha-1} A_{p}^{\beta} a_{p}\right| \leq \max _{1 \leq m \leq v}\left|\sum_{p=0}^{m} A_{m-p}^{\alpha-1} A_{p}^{\beta} a_{p}\right| .
$$

\footnotetext{
*Corresponding author e-mail: hbor33@gmail.com
} 
Lemma 2 ( [6]). Under the conditions (4) and (5), we have $n X_{n}\left|\Delta \lambda_{n}\right|=O(1)$ as $n \rightarrow \infty$,

$$
\sum_{n=1}^{\infty} X_{n}\left|\Delta \lambda_{n}\right|<\infty
$$

\section{Proof of the theorem}

Let $\left(T_{n}^{\alpha, \beta}\right)$ be the $n$th $(C, \alpha, \beta)$ mean, with $0<\alpha \leq 1$ and $\beta>-1$, of the sequence

$\left(n a_{n} \lambda_{n} \varphi_{n}\right)$. Then, by (1), we have that

$T_{n}^{\alpha, \beta}=\frac{1}{A_{n}^{\alpha+\beta}} \sum_{v=1}^{n} A_{n-v}^{\alpha-1} A_{v}^{\beta} v a_{v} \lambda_{v} \varphi_{n}$.

Thus, applying Abel's transformation first and then using Lemma 1, we have that

$$
\begin{aligned}
T_{n}^{\alpha, \beta}= & \frac{1}{A_{n}^{\alpha+\beta}} \sum_{v=1}^{n-1} \Delta\left(\lambda_{v} \varphi_{n}\right) \sum_{p=1}^{v} A_{n-p}^{\alpha-1} A_{p}^{\beta} p a_{p} \\
& +\frac{\lambda_{n} \varphi_{n}}{A_{n}^{\alpha+\beta}} \sum_{v=1}^{n} A_{n-v}^{\alpha-1} A_{v}^{\beta} v a_{v}, \\
= & \frac{1}{A_{n}^{\alpha+\beta}} \sum_{v=1}^{n-1}\left(\lambda_{v} \Delta \varphi_{v}+\varphi_{v+1} \Delta \lambda_{v}\right) \sum_{p=1}^{v} A_{n-p}^{\alpha-1} A_{p}^{\beta} p a_{p} \\
+ & \frac{\lambda_{n} \varphi_{n}}{A_{n}^{\alpha+\beta}} \sum_{v=1}^{n} A_{n-v}^{\alpha-1} A_{v}^{\beta} v a_{v} . \\
\left|T_{n}^{\alpha+\beta}\right| \leq & \frac{1}{A_{n}^{\alpha+\beta}} \sum_{v=1}^{n-1}\left|\lambda_{v} \Delta \varphi_{v}\right|\left|\sum_{p=1}^{v} A_{n-p}^{\alpha-1} A_{p}^{\beta} p a_{p}\right| \\
& +\frac{1}{A_{n}^{\alpha+\beta}} \sum_{v=1}^{n-1}\left|\varphi_{v+1} \Delta \lambda_{v}\right|\left|\sum_{p=1}^{v} A_{n-p}^{\alpha-1} A_{p}^{\beta} p a_{p}\right| \\
& +\frac{\left|\lambda_{n} \varphi_{n}\right|}{A_{n}^{\alpha+\beta}}\left|\sum_{v=1}^{v} A_{n-v}^{\alpha-1} A_{v}^{\beta} v a_{v}\right| \\
\leq & \frac{1}{A_{n}^{\alpha+\beta}} \sum_{v=1}^{n-1} A_{v}^{\alpha+\beta} w_{v}^{\alpha, \beta}\left|\lambda_{v}\right|\left|\Delta \varphi_{v}\right| \\
& +\frac{1}{A_{n}^{\alpha+\beta}} \sum_{v=1}^{n-1} A_{v}^{\alpha+\beta} w_{v}^{\alpha, \beta}\left|\varphi_{v+1}\right|\left|\Delta \lambda_{v}\right| \\
& +\left|\lambda_{n}\right|\left|\varphi_{n}\right| w_{n}^{\alpha, \beta} \\
= & T_{n, 1}^{\alpha, \beta}+T_{n, 2}^{\alpha, \beta}+T_{n, 3}^{\alpha, \beta} .
\end{aligned}
$$

To complete the proof of the theorem, by Minkowski's inequality, it is sufficient to show that

$\sum_{n=1}^{\infty} n^{-1}\left|T_{n, r}^{\alpha, \beta}\right|^{k}<\infty$, for $r=1,2,3$.

When $k>1$, we can apply Hölder's inequality with indices $k$ and $k^{\prime}$, where $\frac{1}{k}+\frac{1}{k^{\prime}}=1$, we get

$$
\begin{aligned}
\sum_{n=2}^{m+1} n^{-1}\left|T_{n, 1}^{\alpha, \beta}\right|^{k} \leq & \sum_{n=2}^{m+1} n^{-1}\left(A_{n}^{\alpha+\beta}\right)^{-k} \times \\
& \left\{\sum_{v=1}^{n-1} A_{v}^{\alpha+\beta} w_{v}^{\alpha, \beta}\left|\Delta \varphi_{v}\right|\left|\lambda_{v}\right|\right\}^{k}
\end{aligned}
$$

$$
\begin{aligned}
& =O(1) \sum_{n=2}^{m+1} \frac{1}{n^{1+(\alpha+\beta) k}} \sum_{v=1}^{n-1}\left(v^{\alpha+\beta}\right)^{k}\left(w_{v}^{\alpha, \beta}\right)^{k}\left|\Delta \varphi_{v}\right|^{k}\left|\lambda_{v}\right|^{k}\left\{\sum_{v=1}^{n-1} 1\right\}^{k-1} \\
& =O(1) \sum_{n=2}^{m+1} \frac{1}{n^{2+(\alpha+\beta-1) k}} \sum_{v=1}^{n-1} v^{(\alpha+\beta) k}\left(w_{v}^{\alpha, \beta}\right)^{k}\left|\lambda_{v}\right|^{k} \frac{1}{v^{k}} \\
& =O(1) \sum_{v=1}^{m} v^{(\alpha+\beta) k}\left(w_{v}^{\alpha, \beta}\right)^{k} v^{-k}\left|\lambda_{v}\right|^{k} \sum_{n=v+1}^{m+1} \frac{1}{n^{2+(\alpha+\beta-1) k}} \\
& =O(1) \sum_{v=1}^{m} v^{(\alpha+\beta) k}\left(w_{v}^{\alpha, \beta}\right)^{k} v^{-k}\left|\lambda_{v}\right|^{k} \int_{v}^{\infty} \frac{d x}{x^{2+(\alpha+\beta-1) k}} \\
& =O(1) \sum_{v=1}^{m}\left(w_{v}^{\alpha, \beta}\right)^{k}\left|\lambda_{v}\right|\left|\lambda_{v}\right|^{k-1} \frac{1}{v} \\
& =O(1) \sum_{v=1}^{m} \frac{\left(w_{v}^{\alpha, \beta}\right)^{k}\left|\lambda_{v}\right|}{v X_{v}^{k-1}} \\
& =O(1) \sum_{v=1}^{m-1} \Delta\left|\lambda_{v}\right| \sum_{r=1}^{v} \frac{\left(w_{r}^{\alpha, \beta}\right)^{k}}{r X_{r}^{k-1}}+O(1)\left|\lambda_{m}\right| \sum_{v=1}^{m} \frac{\left(w_{v}^{\alpha, \beta}\right)^{k}}{v X_{v}^{k-1}} \\
& =O(1) \sum_{v=1}^{m}\left|\Delta \lambda_{v}\right| X_{v}+O(1)\left|\lambda_{m}\right| X_{m}=O(1), m \rightarrow \infty
\end{aligned}
$$

by virtue of the hypotheses of the theorem and Lemma 2 . Again, we get that

$$
\begin{aligned}
& \sum_{n=2}^{m+1} n^{-1}\left|T_{n, 2}^{\alpha, \beta}\right|^{k} \leq \sum_{n=2}^{m+1} n^{-1}\left(A_{n}^{\alpha+\beta}\right)^{-k} \times \\
&\left\{\sum_{v=1}^{n-1} A_{v}^{\alpha+\beta} w_{v}^{\alpha, \beta}\left|\varphi_{v+1}\right|\left|\Delta \lambda_{v}\right|\right\}^{k} \\
&= O(1) \sum_{n=2}^{m+1} \frac{1}{n^{1+(\alpha+\beta) k}}\left\{\sum_{v=1}^{n-1} v^{\alpha+\beta}\left(w_{v}^{\alpha, \beta}\right)\left|\Delta \lambda_{v}\right|\right\}^{k} \\
&= O(1) \sum_{n=2}^{m+1} \frac{1}{n^{1+(\alpha+\beta) k}} \sum_{v=1}^{n-1} \frac{v^{(\alpha+\beta) k}\left(w_{v}^{\alpha, \beta}\right)^{k}\left|\Delta \lambda_{v}\right|}{X_{v}^{k-1}} \times \\
&\left\{\sum_{v=1}^{n-1} X_{v}\left|\Delta \lambda_{v}\right|\right\} \\
&= O(1) \sum_{n=2}^{m+1} \frac{1}{n^{1+(\alpha+\beta) k}} \sum_{v=1}^{n-1} \frac{v^{(\alpha+\beta) k}\left(w_{v}^{\alpha, \beta}\right)^{k}\left|\Delta \lambda_{v}\right|}{X_{v}^{k-1}} \\
&= O(1) \sum_{v=1}^{m} \frac{v^{(\alpha+\beta) k}\left(w_{v}^{\alpha, \beta}\right)^{k}\left|\Delta \lambda_{v}\right|}{X_{v}^{k-1}} \sum_{n=v}^{m+1} \frac{1}{n^{1+(\alpha+\beta) k}} \\
&= O(1) \sum_{v=1}^{m} \frac{v^{(\alpha+\beta) k}\left(w_{v}^{\alpha, \beta}\right)^{k}\left|\Delta \lambda_{v}\right|}{X_{v}^{k-1}} \int_{v=1}^{\infty} \frac{d x}{x^{1+(\alpha+\beta) k}} \\
& v\left|\Delta \lambda_{v}\right| \frac{\left(w_{v}^{\alpha, \beta}\right)^{k}}{v X_{v}^{k-1}}
\end{aligned}
$$

$=O(1) \sum_{v=1}^{m} \Delta\left(v\left|\Delta \lambda_{v}\right|\right) \sum_{r=1}^{v} \frac{\left(w_{r}^{\alpha, \beta}\right)^{k}}{r X_{r}^{k-1}}+O(1) m\left|\Delta \lambda_{m}\right| \sum_{v=1}^{m} \frac{\left(w_{v}^{\alpha, \beta}\right)^{k}}{v X_{v}^{k-1}}$ $=O(1) \sum_{v=1}^{m-1} v\left|\Delta^{2} \lambda_{v}\right| X_{v}+O(1) \sum_{v=1}^{m-1} X_{v}\left|\Delta \lambda_{v}\right|+O(1) m\left|\Delta \lambda_{m}\right| X_{m}$ 
$=O(1)$, as $m \rightarrow \infty$

by hypotheses of the theorem and Lemma 2. Finally, as in $T_{n, 1}^{\alpha, \beta}$, we have that

$$
\begin{aligned}
\sum_{n=1}^{m} n^{-1}\left|T_{n, 3}^{\alpha, \beta}\right|^{k} & =\sum_{n=1}^{m} n^{-1}\left|\lambda_{n} \varphi_{n} w_{n}^{\alpha, \beta}\right|^{k} \\
& =O(1) \sum_{n=1}^{m} \frac{\left(w_{n}^{\alpha, \beta}\right)^{k}\left|\lambda_{n}\right|}{n X_{n}^{k-1}}=O(1) \text {, as } m \rightarrow \infty
\end{aligned}
$$

by virtue of the hypotheses of the theorem and Lemma 2 . This completes the proof of the theorem. It should be noted that, if we take $\beta=0$ and $\alpha=1$, then we get Theorem A. If we take $\beta=0$, then we get a result concerning the $|C, \alpha|_{k}$ summability factors of infinite series. Also, if we take $k=1$ and $\beta=0$, then we get a new result dealing with the $|C, \alpha|$ summability factors of infinite series.

\section{References}

[1] N. K. Bari and S. B. Stečkin, Best approximation and differential properties of two conjugate functions, Trudy. Moskov. Mat. Ob̌̌ ̌̌., 5, 483-522 (1956).

[2] H. Bor, On a new application of power increasing sequences, Proc. Est. Acad. Sci., 57, 205-209 (2008).

[3] D. Borwein, Theorems on some methods of summability, Quart. J. Math. Oxford Ser., 9, 310-316 (1958).

[4] G. Das, A Tauberian theorem for absolute summability, Proc. Camb. Phil. Soc., 67, 321-326 (1970).

[5] T. M. Flett, On an extension of absolute summability and some theorems of Littlewood and Paley, Proc. London Math. Soc., 7, 113-141 (1957).

[6] S. M. Mazhar, Absolute summability factors of infinite series, Kyungpook Math. J., 39, 67-73 (1999).

[7] W. T. Sulaiman, On a new application of almost increasing sequences, Bull. Math. Anal. Appl., 4, 29-33 (2012).

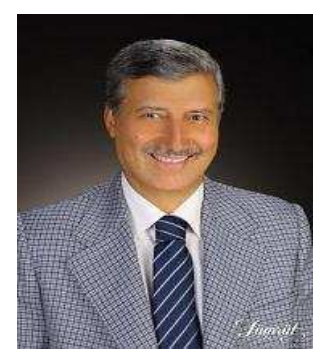

\section{Hüseyin Bor}

received his $\mathrm{PhD}$ degree in Mathematics Department-Faculty of Science, Ankara University (Turkey) in 1982 and he is presently an Emeritus Professor of Mathematical Analysis. His research interests are Classical Analysis (especially summability theory), Fourier Analysis and Special Functions. He is author of more than 195 research papers published in the reputed international mathematics journals. He is also referee and editor of many mathematical journals. 